\title{
On the Continuity of Automorphic Representations of Groups
}

\author{
JOHAN F. AARNES* \\ University of Oslo, Norway \\ Received October 15, 1967
}

\begin{abstract}
Given a weakly continuous automorphic representation $\alpha$ of a group $G$ on a concrete $C^{*}$-algebra $\mathfrak{A}$, we show that a mild joint continuity condition makes it possible to extend $\alpha$ to a weakly continuous representation of $G$ on the weak closure of $\mathfrak{A}$. If $G$ is locally compact and $\mathfrak{A}$ is a von Neumann algebra, this condition is automatically satisfied.
\end{abstract}

\section{Introduction}

Let $G$ be a topological group, and let $\mathfrak{A}$ be a $C^{*}$-algebra with the identity operating on a Hilbert-space $H$. Let $\alpha: G \rightarrow$ aut $(\mathfrak{U})=$ the group of *automorphisms of $\mathfrak{A}$, be a homomorphism, and let $\alpha_{g}$ denote image of $g \in G$ under $\alpha$. We require that the map

$$
A:(g, x) \rightarrow \alpha_{g}(x) ; \quad g \in G, \quad x \in \mathfrak{A}
$$

of $G \times \mathfrak{A}$ into $\mathfrak{A}$ is separately continuous when restricted to $G \times \mathfrak{A}_{1}$, and $\mathfrak{A}_{1}=$ the unit sphere of $\mathfrak{A}$, is given the weak operator topology. In this case we say that $\alpha$ is an automorphic representation of $G$ on $\mathfrak{A}$.

Since $\left(\alpha_{g}\right)^{-1}=\alpha_{g^{-1}}$, it follows in particular that each $\alpha_{g} ; g \in G$, is weakly bi-continuous on the unit sphere of $\mathfrak{A}$, and therefore has an extension to $\overline{\mathfrak{A}}=$ the weak closure of $\mathfrak{A}$ on $H$, as a $*$-automorphism which we again denote by $\alpha_{g}$ ([4], remark 2.2.3). Each $\alpha_{g}$ is weakly bicontinuous on the unit sphere of $\overline{\mathfrak{A}}$, and the map $\bar{\alpha}: G \rightarrow \operatorname{aut}(\overline{\mathfrak{A}})$ obtained this way is a homomorphism. It is a question of some interest to know under what conditions $\bar{\alpha}$ is an automorphic representation of $G$ on $\overline{\mathfrak{A}}$, i.e., when the map $g \rightarrow \alpha_{g}(x) ; x \in \overline{\mathfrak{A}}_{1}$, is weakly continuous. For a motivation, see f.i. Dell'Antonio [2] or Kadrson [5]. It turns out that a mild joint continuity condition on the map $A$ is sufficient, and that this condition is automatically satisfied if $\mathfrak{A}$ is a von Neumann algebra.

\section{Extension of Automorphic Representations}

We say that the automorphic representation $\alpha$ of $G$ on $\mathfrak{A}$ is extendable, of $\bar{\alpha}$ is an automorphic representation of $G$ on $\overline{\mathfrak{A}}$. Clearly, several other topologies on $\mathfrak{A}$ were possible in the definition of an automorphic rep-

\footnotetext{
* Research supported by NSF.
} 
resentation. To clarify matters, we include the following supposedly well-known result:

Lemma 1. Let $\alpha: G \rightarrow$ aut(2) be a homomorphism. The following conditions are equivalent $\}$

(i) A restricted to $G \times \mathfrak{A}_{1}$ is separably continuous when $\mathfrak{A}$ is given the weak operator topology.

(ii) $A$ is separably continuous on $G \times \mathfrak{A}$ when $\mathfrak{A}$ is given the ultraweak operator topology.

(iii) A restricted to $G \times \mathfrak{A}_{1}$ is separately continuous when $\mathfrak{Z}$ is given the strong operator topology.

(iv) $A$ is separably continuous on $G \times \mathfrak{A}$ when $\mathfrak{A}$ is given the ultrastrong operator topology.

Proof. Each of the four conditions imply that each $\alpha_{g} ; g \in G$, extends to a *automorphism of $\overline{\mathfrak{A}}$. The continuity of $A$ for fixed $g \in G$ in all the four cases above then follows from cor 1, p. 57 [3]. Next, we show that the requirement of continuity of the map $g \rightarrow \alpha_{g}(x) ; x \in \mathfrak{A}$ fixed, is the same in the four topologies mentioned above. To do this, we recall that the strong and ultrastrong operator topologies coincide on bounded sets, and that the weak and ultrastrong topology is the weakest resp. strongest of the lot. So, we only have to prove that for an arbitrary $x$, which we may take in $\mathfrak{A}_{1}$, weak continuity of $g \rightarrow \alpha_{g}(x)$ implies strong continuity. Let $g_{v} \rightarrow g$ in $G$, and let $\xi \in H$ be arbitrary.

$$
\begin{aligned}
\left\|\left(\alpha_{g_{\nu}}(x)-\alpha_{g}(x)\right) \xi\right\|^{2}= & \left(\left(\alpha_{g_{\nu}}(x)-\alpha_{g}(x)\right) *\left(\alpha_{g_{v}}(x)-\alpha_{g}(x)\right) \xi, \xi\right) \\
= & \left(\alpha_{g_{v}}\left(x^{*} x\right) \xi, \xi\right)-\left(\alpha_{g_{\nu}}\left(x^{*}\right) \alpha_{g}(x) \xi, \xi\right) \\
& -\left(\alpha_{g}\left(x^{*}\right) \alpha_{g_{\nu}}(x) \xi, \xi\right)+\left(\alpha_{g}\left(x^{*} x\right) \xi, \xi\right)
\end{aligned}
$$

which by assumption converges to 0 . This proves the lemma.

Since it will be convenient, we shall assume that $\mathfrak{A}$ is given the (relativized) ultraweak operator topology. For short, we denote this topology by $(u w)$. Let $\bar{A}$ denote the extension of $A$ to $G \times \overline{\mathfrak{A}}$ into $\overline{\mathfrak{A}}$. Clearly $\alpha$ is extendable if and only if $\bar{A}$ is separately continuous. Let $e$ be the unit of $G$, and let $\mathfrak{A}_{1}^{+}$be the positive part of $\mathfrak{A}_{1}$.

Lemma 2. Suppose that $A$ is jointly continuous at $(e, 0)$ when restricted to $G \times \mathfrak{A}_{1}^{+}$. Then $\bar{A}$ is jointly continuous at $(e, 0)$ when restricted to $G \times \overline{\mathfrak{A}}_{1}^{+}$.

Proof. Let $\varepsilon>0$ and a normal state $\varphi$ on $\overline{\mathfrak{I}}$ be given. By assumption there is a neighborhood $U$ of $e$ in $G$, and a normal state $\psi$ on $\overline{\mathfrak{A}}$ and $\delta>0$ such that if $g \in U$ and $x \in \mathfrak{Q}_{1}^{+}, \psi(x)<\delta$, then $\varphi\left(\alpha_{g}(x)\right)<\varepsilon$. Now suppose $x_{0} \in \overline{\mathfrak{Q}}_{1}^{+}$and $\psi\left(x_{0}\right)<\delta$. We have for $x \in \mathfrak{P}_{1}^{+}$:

$$
\varphi\left(\alpha_{g}\left(x_{0}\right)\right)=\varphi\left(\alpha_{g}\left(x_{0}\right)-\alpha_{g}(x)\right)+\varphi\left(\alpha_{g}(x)\right) .
$$

Since $\mathfrak{Q}_{1}^{+}$is $(u w)$-dense in $\overline{\mathfrak{A}}_{1}^{+}$(the Kaplansky density theorem), we can find a net $\left\{x_{\gamma}\right\} \cong \mathfrak{A}_{1}^{+}$such that $x_{\gamma} \rightarrow x_{0}(u w)$. Since $\psi\left(x_{0}\right)<\delta$, we know 
that $\psi\left(x_{\gamma}\right)<\delta$ eventually, so that $\varphi\left(\alpha_{g}\left(x_{\gamma}\right)\right)<\varepsilon$. Moreover, $\alpha_{g}$ is $(u w)$ continuous, so $\varphi\left(\alpha_{g}\left(x_{0}\right)-\alpha_{g}\left(x_{\gamma}\right)\right) \rightarrow 0$, which proves that $\varphi\left(\alpha_{g}\left(x_{0}\right)\right)<\varepsilon$. This proves the lemma.

Lemma 3. Suppose that $\bar{A}$ is jointly continuous at $(e, 0)$ when restricted to $G \times \overline{\mathfrak{A}}_{1}^{+}$. Then $\alpha$ is extendable, i.e. for each $x \in \overline{\mathfrak{A}}$, the map $g \rightarrow \alpha_{g}(x)$ is (uw)-continuous.

Proof. Without loss of generality, we may take $x \geqq 0,\|x\| \leqq 1 / 2$, i.e., $x \in \overline{\mathfrak{Q}}_{1 / 2}^{+}$. Let $\varepsilon>0$ and the normal state $\varphi$ be given, choose $U$ around $e$ in $G$, a normal state $\psi$ and $\delta>0$ such that $g \in U$ and $y \in \overline{\mathfrak{A}}_{1}^{+} \cap V$, with $V=\{y \in \overline{\mathfrak{U}}:|\psi(y)|<\delta\}$, implies $\alpha_{g}(y) \in \overline{\mathfrak{A}}_{1}^{+} \cap W$, with $W=\{z \in \overline{\mathfrak{U}}:|\varphi(z)|<\varepsilon\}$. $\mathfrak{A}_{1 / 2}$ is ultrastrongly dense in $\overline{\mathfrak{A}}_{1 / 2}$, so we can find an element $x_{0} \in \mathfrak{A}_{1 / 2}$ such that $\psi\left(\left(x_{0}-x\right)^{*}\left(x_{0}-x\right)\right)<\delta$, i.e., $y_{0}=\left(x_{0}-x\right)^{*}\left(x_{0}-x\right) \in \overline{\mathfrak{A}}_{1}^{+} \cap V$, since $\left\|y_{0}\right\|=\left\|x_{0}-x\right\|^{2} \leqq 1$ and $y_{0} \geqq 0$. The map $g \rightarrow \alpha_{g}\left(x_{0}\right)$ is $(u w)$ continuous since $x_{0} \in \mathfrak{A}$, so there is a neighborhood $U^{\prime}$ of $e$ in $G$ such that $g \in U^{\prime}$ implies $\alpha_{g}\left(x_{0}\right)-x_{0} \in W ; g \in G$. Hence

$$
\begin{aligned}
\left|\varphi\left(\alpha_{g}(x)-x\right)\right| & \leqq\left|\varphi\left(\alpha_{g}(x)-\alpha_{g}\left(x_{0}\right)\right)\right| \\
& +\left|\varphi\left(\alpha_{g}\left(x_{0}\right)-x_{0}\right)\right|+\left|\varphi\left(x_{0}-x\right)\right| \\
& \leqq \varphi\left(\alpha_{g}\left(y_{0}\right)\right)^{1 / 2}+\left|\varphi\left(\alpha_{g}\left(x_{0}\right)-x_{0}\right)\right|+\varphi\left(y_{0}\right)^{1 / 2}
\end{aligned}
$$

so if $g \in U \cap U^{\prime}$ it follows that $\alpha_{g}\left(y_{0}\right)$ and $y_{0}=\alpha_{e}\left(y_{0}\right)$ both belong to $W \cap \overline{\mathfrak{A}}_{1}^{+}$, and $\left|\varphi\left(\alpha_{g}(x)-x\right)\right|<\varepsilon^{1 / 2}+\varepsilon+\varepsilon^{1 / 2}$. This proves that $g \rightarrow \alpha_{g}(x)$ is $(u w)$-continuous at $e \in G$.

Now let $g_{0} \in G$ be arbitrary. Choose a neighbourhood $U^{\prime \prime}$ of $e$ in $G$ such that $g \in U^{\prime \prime}$ implies $\alpha_{g}\left(\alpha_{g_{0}}(x)\right)-\alpha_{g_{0}}(x) \in W$. Then, for $h g_{0}^{-1} \in U^{\prime \prime}$ we have

$$
\alpha_{h}(x)-\alpha_{g_{0}}(x)=\alpha_{h g_{0}^{-1}}\left(\alpha_{g_{0}}(x)\right)-\alpha_{g_{0}}(x) \in W,
$$

which proves continuity in general. The proof is complete.

The conclusion to draw from the two preceding lemmas is of course that if $A$ is jointly continuous at $(e, 0)$ when restricted to $G \times \mathfrak{A}_{1}^{+}$, then $\alpha$ is extendable. However, much more can be said in the case when $G$ is locally compact. Let $N$ denote the pre-dual of $\overline{\mathfrak{A}}$, and let $S_{n}$ be the convex set of normal states on $\overline{\mathfrak{A}}$. For each $g \in G$ we may define a map $v_{g}$ of $S_{n}$ onto itself by $v_{g}(\varphi)(x)=\varphi\left(\alpha_{g}(x)\right) ; \varphi \in S_{n}, x \in \mathfrak{A}$. Each $v_{g}$ is an affine unimorphism on $S_{n}$ when $S_{n}$ is given the relative $w^{*}$-topology $\sigma\left(\mathfrak{Z}^{*}, \mathfrak{Z}\right)$ (see [4]). Moreover, for each $\varphi \in S_{n}$, the map $g \rightarrow v_{g}(\varphi)$ is $w^{*}$-continuous.

Theorem. Let $G$ be locally compact, and let $\alpha$ be an automorphic representation of $G$ on $\mathfrak{A}$. The following conditions are equivalent.

(i) $\alpha$ is extendable.

(ii) $g \rightarrow v_{g}(\varphi)$ is $\sigma(N, \overline{\mathfrak{X}})$-continuous for each $\varphi \in S_{n}$.

(iii) For each compact set $U \leqq G$, and each $\varphi \in S_{n}$, the orbitpiece $\left\{\boldsymbol{v}_{g}(\varphi): g \in U\right\}$ is $\sigma(N, \overline{\mathfrak{A}})$-compact. 
(iv) For any compact set $U \leqq G$, the family $\left\{\alpha_{g}: g \in U\right\}$ is $(u w)$ equicontinuous at 0 when restricted to $\overline{\mathfrak{A}}_{1}^{+}$.

(v) $A$ is jointly continuous at $(e, 0)$ when restricted to $G \times \mathfrak{A}_{1}^{+}$.

Proof. (i) $\Rightarrow$ (ii). For $g \in G$ and $\varphi \in S_{n}$ we have $v_{g}(\varphi)(x)=\varphi\left(\alpha_{g}(x)\right)$ for all $x \in \mathfrak{A}$ by definition. $\varphi$ is normal and $\alpha_{g}$ is $(u w)$-continuous so this equality holds for all $x \in \overline{\mathfrak{A}}$. If $\alpha$ is extendable it therefore follows that $g \rightarrow v_{g}(\varphi)$ is $\sigma(N, \overline{\mathfrak{A}})$-continuous of $G$ into $S_{n}$ for each $\varphi$.

(ii) $\Rightarrow$ (iii). Obvious.

(iii) $\Rightarrow$ (iv). Let a compact set $U \leqq G$ be given, let $\varepsilon>0$ and $\varphi$ an arbitrary normal state. By assumption the set $\left\{\nu_{g}(\varphi): g \in U\right\}$ is $\sigma(N, \overline{\mathfrak{U}})$ compact. By theorem $1,[1]$, there is a normal state $\psi$ and a number $\delta>0$ such that if $x \in \overline{\mathfrak{A}}_{1}$ and $\psi\left(x^{*} x+x x^{*}\right)<\delta$, then $\left|v_{g}(\varphi)(x)\right|<\varepsilon$ for all $g \in U$. Hence, if $x \in \overline{\mathfrak{Q}}_{1}^{+}$and $\psi(x)<\frac{\delta}{2}$ we get

$$
\psi\left(x^{*} x+x x^{*}\right)=2 \psi\left(x^{2}\right) \leqq 2 \psi(x)<\delta
$$

so $\left|\varphi\left(\alpha_{g}(x)\right)\right|=\left|\nu_{g}(\varphi)(x)\right|<\varepsilon$ for all $g \in U$. This proves the equicontinuity of $\alpha_{g} \mid \overline{\mathfrak{A}}_{1}^{+}$at 0 .

(iv) $\Rightarrow(v)$. Let the normal state $\varphi$ and $\varepsilon>0$ be given. Choose an arbitrary compact neighbourhood $U$ of $e$ in $G$. The family $\left\{\alpha_{g} \mid \overline{\mathfrak{A}}_{1}^{+}: g \in U\right\}$ is then equicontinuous at 0 , so there is a normal state $\psi$ and $\delta>0$ such that $g \in U, x \in \overline{\mathfrak{A}}_{1}^{+}$and $\psi(x)<\delta$ implies $\left|\varphi\left(\alpha_{g}(x)\right)\right|<\varepsilon$. This proves $(\mathrm{v})$, and the implication $(\mathrm{v}) \Rightarrow(\mathrm{i})$ is already proved.

Note. Only the proof of the implication (iv) $\Rightarrow(\mathrm{v})$ makes use of the assumption that $G$ is locally compact.

Corollary. If $\alpha$ is an automorphic representation of a locally compact group $G$ on a von Neumann algebra $\mathfrak{A}$, then $A$ is jointly continuous at $(e, 0)$ when restricted to $G \times \mathfrak{A}_{1}^{+}$

\section{Joint Continuity of Automorphic Representations}

If $\alpha$ is an automorphic representation of a topological group $G$ on a concrete $C^{*}$-algebra $\mathfrak{A}$, it is of some interest to see how far joint continuity of the map $A: G \times \mathfrak{A} \rightarrow \mathfrak{A}$ extends, once we have assumed it at $(e, 0)$. To answer this question satisfactorily, we have to switch to the ultrastrong operator topology on $\mathfrak{A}$, which we abbreviate to $(u s)$. The $(u s)$-topology is determined by the family of semi-norms $\|z\|_{\varphi}=\varphi\left(z^{*} z\right)^{1 / 2}$; $z \in \mathfrak{A}$, where $\varphi$ runs thru $S_{n}$. We first prove the following lemma.

Lemma 4. Let $\alpha$ be an extendable automorphic representation of $G$ on $\mathfrak{A}$. Then, for each compact set $U \leqq G$ and each bounded set $B \leqq \mathfrak{A}$, the family $\left\{\alpha_{g}: g \in U\right\}$ is uniformly (us)-equicontinuous when restricted to $B$.

Proof. We must show that for any $\varphi \in S_{n}, \varepsilon>0$ given, there exists $\psi \in S_{n}, \delta>0$ such that if $x, y \in B$ and $\|x-y\|_{\psi}<\delta$, then $\left\|\alpha_{g}(x)-\alpha_{g}(y)\right\|_{\varphi}<\varepsilon$ for all $g \in U$. So let $\varphi, \varepsilon>0$ be given, and suppose that the diameter 23 Commun. math. Phys., Vol. 7 
of $B$ is less than $M>1$. By (iv) in the theorem, we can find $\psi \in S_{n}$, $\delta>0$ such that if $z \in \mathfrak{A}_{1}^{+}$and $\psi(z)<\delta$, then $\varphi\left(\alpha_{g}(z)\right)<\frac{\varepsilon^{2}}{M^{2}}$ for all $g \in U$. Now let $x, y \in B$ and suppose that $\|x-y\|_{\psi}<\delta^{1 / 2}$. Put $y^{\prime}$ $=x+\frac{1}{M}(y-x)$, so $\left\|y^{\prime}-x\right\| \leqq 1$. Then $z=\left(y^{\prime}-x\right)^{*}\left(y^{\prime}-x\right) \in \mathfrak{A}_{1}^{+}$, and $\psi(z)=\left\|y^{\prime}-x\right\|_{\psi}^{2}=\frac{1}{M^{2}}\|y-x\|_{\psi}^{2}<\frac{\delta}{M^{2}}<\delta$, so $\left\|\alpha_{g}(y)-\alpha_{g}(x)\right\|_{\varphi}$ $=\left\|\alpha_{g}(y-x)\right\|_{\varphi}=M\left\|\alpha_{g}\left(y^{\prime}-x\right)\right\|_{\varphi}=M \varphi\left(\alpha_{g}(z)\right)^{1 / 2}<\varepsilon$ for all $g \in U$. The proof is complete.

Corollary. Let $\alpha$ be an automorphic representation of a compact group $G$ on a von Neumann algebra $\mathfrak{A}$. Then $\left\{\alpha_{g}: g \in G\right\}$ is uniformly (us)equicontinuous on each bounded set $B \leqq \mathfrak{A}$.

Proposition. Let $\alpha$ be an extendable automorphic representation of a locally compact group $G$ on a $C^{*}$-algebra $\mathfrak{A}$. Then $A$ restricted to $G \times B$ is jointly (us)-continuous for each bounded set $B \leqq \mathfrak{A}$.

Proof. Let the bounded set $B \subseteq \mathfrak{A}$ be given, and choose an arbitrary point $(g, x) \in G \times B$. Let $\varphi \in S_{n}, \varepsilon>0$ be given, and choose a compact neighbourhood $U$ of $g$ in $G$ such that $\left\|\alpha_{h}(x)-\alpha_{g}(x)\right\|_{\phi}<\frac{\varepsilon}{2}$ for $h \in U$ (lemma 1). Then, by lemma 4 , there exists $\psi \in S_{n}, \delta>0$ such that if $y \in B$ and $\|x-y\|_{\psi}<\delta$, we get $\left\|\alpha_{h}(y)-\alpha_{h}(x)\right\|_{\varphi}<\frac{\varepsilon}{2}$ for all $h \in U$. Consequently, $\left\|\alpha_{h}(y)-\alpha_{g}(x)\right\|_{\varphi} \leqq\left\|\alpha_{h}(y)-\alpha_{h}(x)\right\|_{\varphi}+\left\|\alpha_{h}(x)-\alpha_{g}(x)\right\|_{\varphi}<\varepsilon$ if $y \in B,\|x-y\|_{\psi}<\delta$ and $h \in U$. The proof is finished.

\section{References}

1. Aarnes, J. F.: On the Mackey-topology for a von Neumann algebra. To appear in Math. Scand.

2. Dell'Antonio, G. F.: On some groups of automorphisms of physical observables. Commun. Math. Phys. 2, 384-397 (1966).

3. Dixmier, J.: Les algebres d'operateurs dans l'espace Hilbertien, Paris: Herrmann 1957.

4. Kadison, R. V.: Unitary invariants for representations of operator algebras. Ann. Math. 66, 304-379 (1957).

5. - Transformation of states in operator theory and dynamics. Vol. 3, p. 177 to 198.1965.

Dr. J. F. AARNES

Department of Mathematics

University of Pennsylvania

Philadelphia, Pa., 19104 USA 